\title{
Thrombus Characteristics Are Related to Collaterals and Angioarchitecture in Acute Stroke
}

\author{
Emmad M. Qazi, Sung Il Sohn, Sachin Mishra, Mohammed A. Almekhlafi, \\ Muneer Eesa, Christopher D. d'Esterre, Abdul A. Qazi, Josep Puig, Mayank Goyal, \\ Andrew M. Demchuk, Bijoy K. Menon
}

\begin{abstract}
Background: We have theorized that clots with stasis are longer. We therefore explored the relationship between thrombus imaging characteristics on noncontrast computed tomography (NCCT) and magnetic resonance imaging (MRI) with clot length and pial collaterals on baseline computed tomography angiography (CTA). Methods: Prospective study of acute ischemic stroke patients (2005-2009) from Keimyung University. Patients with known stroke symptom onset time, baseline CTA, MRI, and with M1-Middle Cerebral Artery $(\mathrm{MCA}) \pm$ intracranial internal carotid artery (ICA) occlusions were included. Clot length and pial collaterals were measured on baseline CTA. Results: A total of 104 patients (mean age $65.1 \pm 12.28$ years, 56.7\% male, median baseline National Institutes of Health Stroke Scale 13) with intracranial ICA + MCA $(n=50)$ or isolated M1-MCA $(n=54)$ occlusions were included. Hyperdense sign on NCCT had a median clot length of $42.3 \mathrm{~mm}$ versus $29.5 \mathrm{~mm}$ when hyperdense negative $(\mathrm{p}=0.02)$. Clots showing blooming artifact on gradient recall echo MRI had a median length of $39.1 \mathrm{~mm}$ versus $24.5 \mathrm{~mm}$ without blooming $(\mathrm{p}=0.005)$. Patients with poor baseline collaterals on CTA had longer clots than those with intermediate/good collaterals (median clot length $49.4 \mathrm{~mm}$ vs $34.9 \mathrm{~mm}$ vs $20.5 \mathrm{~mm}$ respectively, $\mathrm{p}<0.001)$. In censored logistic regression modeling, clot length was an independent predictor of hyperdense sign $(p=0.05)$ and of the presence of blooming artifact $(p=0.006)$. Conclusions: Clot length and baseline collateral status are independent predictors of clot hyperdensity on NCCT and blooming artifact on gradient recall echo. Longer clots are more likely to be hyperdense and to bloom more, probably because portions of these clots are freshly formed locally due to of stasis of blood around the original clot. This stasis could be because of poor collaterals and inefficient angio-architecture within the cerebral arterial tree.
\end{abstract}

RÉSUMÉ: Les caractéristiques du thrombus sont associées aux collatérales et à l'architecture vasculaire dans l'accident vasculaire cérébral aigu. Contexte: Nous avons émis l'hypothèse que les caillots avec stase sont plus longs. Nous avons donc exploré la relation entre les caractéristiques du caillot, à l'imagerie par tomodensitométrie (CT) sans agent de contraste (CTSAC) et à l'imagerie par résonance magnétique (IRM), et la longueur du caillot et les vaisseaux collatéraux pie-mériens à l'angiographie CT initiale (CTA). Méthode: Cette étude prospective porte sur des patients ayant subi un accident vasculaire cérébral ischémique entre 2005 et 2009 qui ont consulté à l'Université Keinyung. Les patients dont le moment du début des symptômes d'AVC était connu, dont le CTA initial et l'IRM étaient au dossier et qui présentaient une occlusion de l'artère cérébrale moyenne M1 (ACM) \pm de la carotide interne intracrânienne (CIIC), ont été inclus dans l'étude. La longueur du caillot et les collatérales pie-mériennes ont été mesurées sur le CTA initial. Résultats: Cent quatre patients, dont l'âge moyen était de 65,1 ans $\pm 12,28$ ans et dont 56,7\% étaient des hommes, ont été inclus dans l'étude. Ils présentaient une occlusion de la CIIC avec occlusion de l'ACM-M1 $(\mathrm{n}=50)$ ou une occlusion isolée de l'ACM-M1 ( $\mathrm{n}=54)$. La longueur médiane du caillot était de 42,3 mm lorsqu'il y avait présence de signe d'hyperdensité au CTSAC et de 29,5 mm lorsqu'il n'y en avait pas ( $\mathrm{p}=0,02)$. Les caillots ayant l'aspect d'un artéfact à l'aspect efflorescent (blooming artifact) à l'IRM gradient recall echo avaient une longueur médiane de 39,1 mm par rapport à 24,5 mm pour ceux qui ne présentaient pas cet aspect $(\mathrm{p}=0,005)$. Les patients qui avaient des collatérales médiocres au CTA initial avaient des caillots plus longs que ceux qui avaient des collatérales intermédiaires ou de bonnes collatérales (longueur médiane du caillot 49,4 mm par rapport à 34,9 mm et 20,5 respectivement, $\mathrm{p}<0,001)$. Le modèle de régression logistique censuré a permis de constater que la longueur du caillot était un facteur de prédiction indépendant de la présence du signe d'hyperdensité $(\mathrm{p}=0,05)$ et de la présence d'un artéfact efflorescent $(\mathrm{p}=0,006)$. Conclusions: La longueur du caillot et l'état initial des collatérales sont des facteurs de prédiction indépendants de l'hyperdensité du caillot au CTSAC et d'un artéfact à l'aspect efflorescent au gradient recall echo. Les caillots plus longs sont plus susceptible d'être hyperdense et de présenter plus d'efflorescence, probablement parce que la formation locale de certaines parties de ces caillots est récente à cause de la stase du sang autour du caillot original. Cette stase pourrait être due à des collatérales médiocres et à une architecture vasculaire inefficace du réseau artériel cérébral.

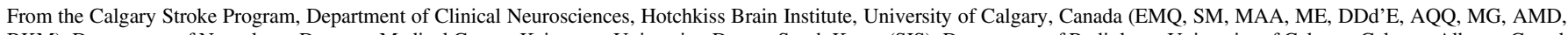

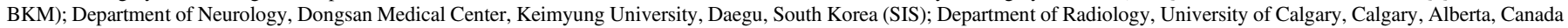
(MAA, ME, MG, AMD, BKM); Faculty of Medicine, King Abdulaziz University, Jeddah, Saudi Arabia (MAM); Department of Radiology, Girona Biomedical Research Institute,

Diagnostic Imaging Institute, Girona, Spain (JP); Department of Community Health Sciences, University of Calgary, Calgary, Alberta, Canada (BKM).

Received June 20, 2014. Final Revisions Submitted June 9, 2015.

Correspondence to: Bijoy K. Menon, 1079 A, 29th Street NW, Calgary, AB, T3H4J2 Canada. Email: Bijoy.Menon@Albertahealthservices.ca 
Keywords: Magnetic Resonance Imaging, stroke, stroke imaging

doi:10.1017/cjn.2015.291

Can J Neurol Sci. 2015; 42: 381-388

The hyperdense sign on noncontrast computed tomography (NCCT) and blooming artifact on gradient recalled echo (GRE) are known to be markers of clot within the cerebral arterial tree. ${ }^{1-8}$ Previous studies have tried to correlate these imaging characteristics with the primary source of clot formation (cardiac vs large artery or in-situ), albeit with varying results. ${ }^{2,3,5,9}$ There is evidence to show that clots that are red blood cell (RBC) rich are hyperdense on NCCT. ${ }^{2,4}$ They also tend to bloom more on GRE because of increased deoxy-hemoglobin content. ${ }^{2,10,11}$

We hypothesize that clot within the cerebral arterial tree consists of two components: (1) the "original clot" that comes either from a proximal source or is formed in-situ due to luminal disease and (2) new clot formation resulting from stasis of blood flow around this "original clot" (Figure 1). In accordance with this hypothesis, clots with significant stasis around the original clot would be longer on imaging (activation of coagulation cascade because of stasis of blood resulting in in situ clot formation). This new clot formation around original clot is potentially $\mathrm{RBC}$-rich because it forms in a low-pressure environment both proximal and distal to the original clot that is lodged in the cerebral arterial tree. The new RBC-rich clot would therefore be hyperdense on NCCT and show blooming on GRE. We reasoned that the degree of stasis and the extent of this new clot formation could depend on two factors: (1) the extent of native collaterals distal to the original clot and (2) the pattern of branching arteries proximal and distal to the clot. Patients with poor collaterals and absence of branching arteries near the original clot would have increased stasis, whereas patients with good collaterals and presence of branching arteries near the original clot would have less stasis (Figure 1).

To test our theory, we sought to determine if clots that are hyperdense on NCCT or have blooming artifact on GRE are longer on computed tomography angiography (CTA), and if longer clots are associated with poorer baseline collaterals. It is also possible that clots change characteristics over time. We therefore tested the hypothesis that the later a clot is imaged, the higher the likelihood that it will not be hyperdense on NCCT/ not bloom on GRE. Based on the results of these investigations, we propose a novel theory of clot formation within the cerebral arterial tree. Finally, we propose a theoretical explanation as to why recanalization rates with intravenous tissue plasminogen activator (tPA) and intra-arterial therapy in patients with acute ischemic stroke may be significantly influenced by the patients' native collateral status and cerebral arterial tree angio-architecture.

\section{METHODS}

The data are from the Keimyung Stroke Registry, an ongoing single-center prospectively collected dataset of patients with acute ischemic stroke presenting to the Keimyung University Hospital, Daegu, South Korea. ${ }^{12,13}$ All patients underwent NCCT at admission followed by a CTA of the head and neck. Most patients also had a baseline magnetic resonance imaging (MRI) scan. Information on demographic and clinical characteristics, medical history, and admission physical examination findings were collected at baseline. Interval times from stroke symptom onset to presentation in the emergency room and imaging were also collected. The local institutional review board approved the study.

\section{Imaging Protocol and Analyses}

Standard nonhelical NCCT was performed on a multislice scanner (Siemens Medical Systems, Forchheim, Germany) with 5-mm slice thickness. NCCT was followed by CTA with a helical scan technique. Coverage was from arch to vertex, with continuous axial slices parallel to the orbitomeatal line with 0.6- to $1.25-\mathrm{mm}$ slice thickness. Acquisitions were obtained after a single bolus intravenous contrast injection of 90 to $120 \mathrm{ml}$ of nonionic contrast media into an antecubital vein at 3-5 $\mathrm{ml} / \mathrm{second}$, autotriggered by appearance of contrast in a region of interest manually placed in the ascending aorta. MRIs were performed with 3 Tesla equipment (Signa Excite, General Electronic, Milwaukee, WI).

The MRI protocol for acute stroke includes diffusion-weighted imaging (DWI), perfusion-weighted imaging (PWI), Fluid-attenuated inversion recovery (FLAIR), apparent diffusion coefficient (ADC) apparent diffusion coefficient, GRE, T1-enhanced image, T1 contrast-enhanced image, and magnetic resonance angiography by the noncontrast-enhanced time-of-flight (TOF) technique. The MRI parameters are as follows: slice thickness of DWI, GRE, and FLAIR image: $5 \mathrm{~mm}$, interslice gap: $2 \mathrm{~mm}$; DWI (repetition time [TR] $6400 \mathrm{msec}$, echo time [TE] $72.4 \mathrm{msec}$ and matrix number $160 \times 160$ ); GRE (TR $550 \mathrm{msec}$, TE $20 \mathrm{~ms}$, flip angle $15^{\circ}$, matrix number $512 \times 160$ ); FLAIR (TR $8000 \mathrm{msec}$, TE $135 \mathrm{msec}$, matrix number $256 \times 160$ ); susceptibility-weighted imaging (TR $40 \mathrm{msec}$, TE $23 \mathrm{msec}$, matrix number $512 \times 192$ and flip angle of $20^{\circ}$ ); and magnetic resonance angiography (TR $18 \mathrm{msec}$, TE of $3.4 \mathrm{msec}$, slice thickness $1.2 \mathrm{~mm}$, interslice gap $1 \mathrm{~mm}$, and matrix number $320 \times 160$ ). For CTA, multiplanar reconstruction and volume rendering was used to reconstruct 24-mm-thick slab maximum intensity projection images in the axial, coronal, and sagittal planes. Baseline and follow-up imaging was analyzed at the imaging core laboratory of the Seaman MR center and the Calgary Stroke Program.

\section{Study Population}

We included only patients presenting with acute ischemic stroke with M1 segment middle cerebral artery (MCA) \pm intracranial internal carotid artery (ICA) occlusions on baseline CTA between May 2004 and July 2009 in the study. This period is relevant because we had access to NCCT, CTA, and MRI at baseline. During this period, there were 286 patients with anterior circulation occlusions on baseline CTA. After excluding 43 patients with MCA occlusions beyond the M1-MCA segment, 28 patients with isolated ICA without M1-MCA occlusion, 


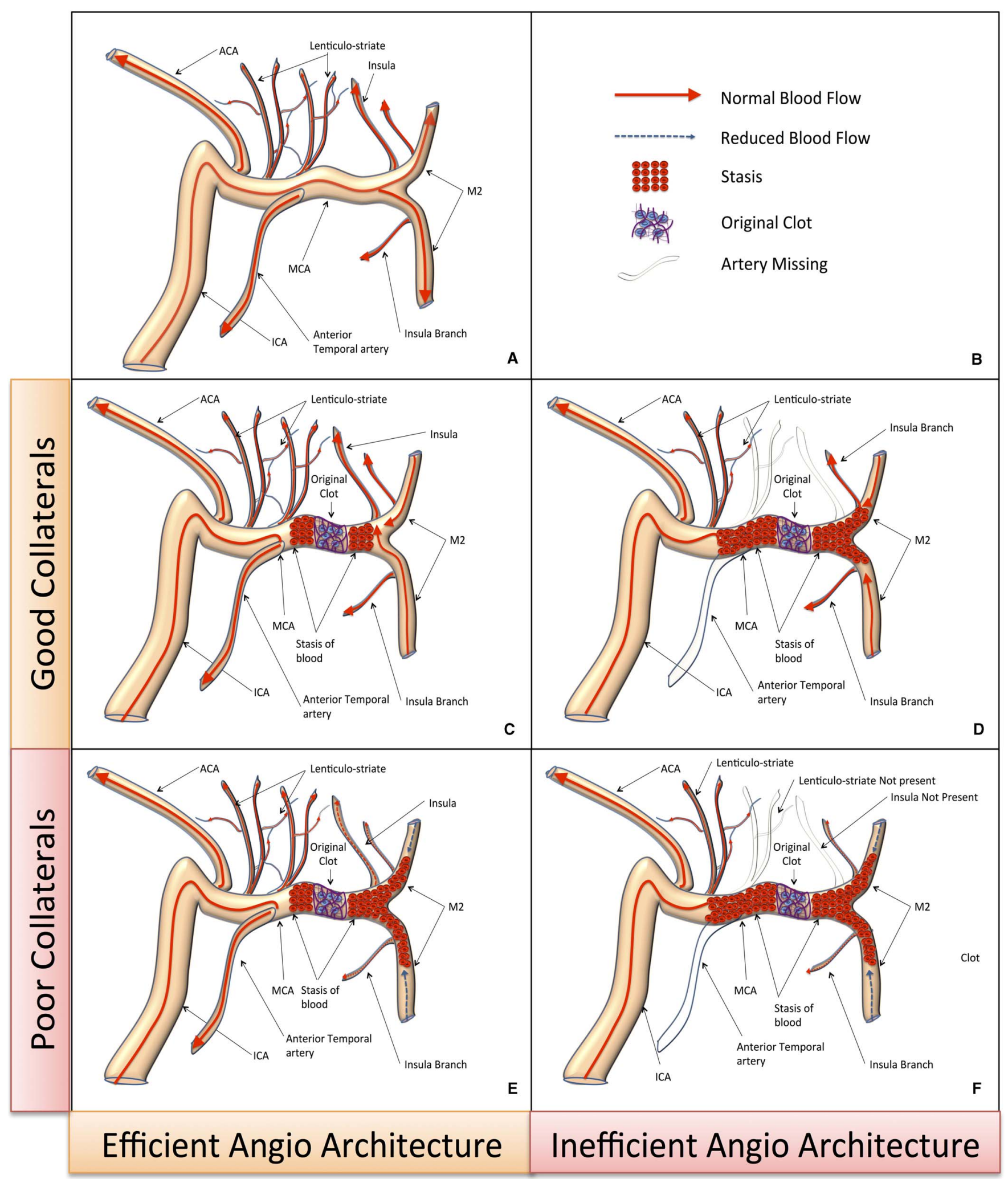

Figure 1: Clot length depends on the extent of pial collaterals and on angio-architecture (branching pattern) around the original clot. (A) Panel A depicts a typical ICA MCA cerebral arterial tree. (C-F) Panel C-F show varying clot lengths according to variability in pial collateral status and angioarchitecture around original clot (B; legends). (C) Panel $C$ depicts a short overall clot length with minimal stasis around original clot (blue) from good collaterals and efficient branching patterns. (D) Panel D depicts longer clot resulting from inefficient angio-architecture (see absence of branching arteries around original clot; dotted vessels) despite good collaterals. (E) Panel E shows a longer clot predominantly because of poor collaterals. $(F)$ Panel $F$ shows very long clots because of poor collaterals and inefficient angio-architecture (absence of branching arteries around original clot). 


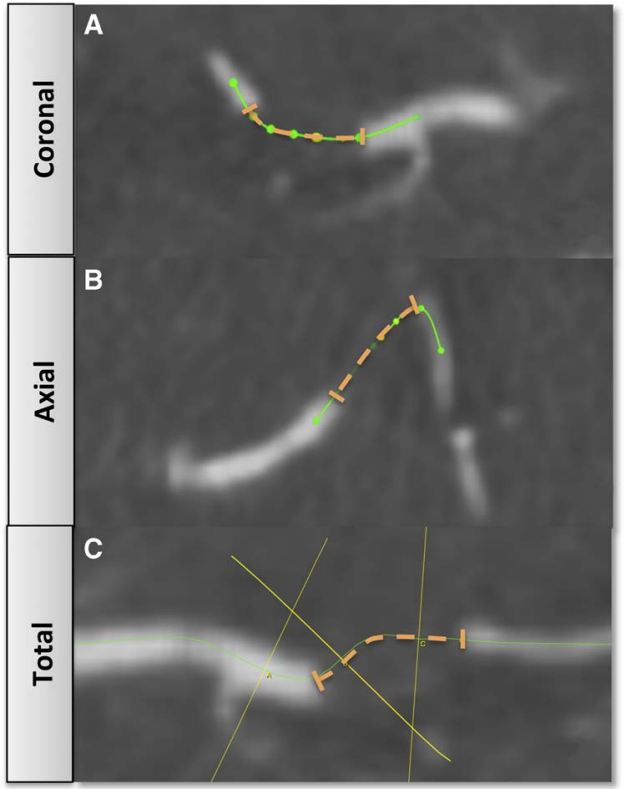

Figure 2: (A-C) Clot length measurement methodology. A line profile was drawn using the three-dimensional multiplanar tool in OsiriX though the clot (green line) using the axial, coronal, and sagittal views. The line profile was used to generate a map that showed the proximal and distal portions of the clot in one plane $(C$ : total). Clot length was measured on axial, coronal, sagittal, and total images. The longest clot length was used as true clot length because it accounted for all curves of the artery.

71 with unknown time of onset of stroke symptom, 16 with baseline MRI obtained posttreatment, 21 with poor-quality MRI at baseline, and 3 with early arterial-weighted baseline CTA, 104 patients were included for analyses.

The presence of the hyperdense sign on NCCT and blooming artifact on GRE was determined by consensus between two stroke neurologists (BKM, SIS). Both readers were blinded to all clinical information and follow-up data at the time of reading the scans. Another stroke neurologist (SM) measured clot length on CTA using OsiriX, version 4. The proximal and distal ends of clots were identified and clot length measured using a tracer tool that traces the profile of the clot within the arterial tree in the axial, sagittal, and coronal planes. The longest length on any of these planes was considered the length of the clot (Figure 2). To increase precision in measuring clot length, we only included patients with M1-MCA \pm intracranial ICA occlusions in this study as clot length could be easily measured in the axial and coronal planes. ${ }^{1}$ We also excluded patients with early arterial-weighted CTAs $(n=3)$ to avoid mislabeling collateral status and the distal clot interface-in early arterial-weighted CTA studies, the contrast may not have had enough time to reach the distal portion of the clot, and as such clot length could be overestimated. Any M1 MCA \pm intracranial ICA clot where the proximal or distal end could not be measured (and therefore were very long) had length imputed to $50 \mathrm{~mm}$. Leptomeningeal collaterals were assessed on baseline CTA by consensus (BKM, SIS) using the regional leptomeningeal score; a previously published ordinal scoring system based on the Alberta Stroke Program Early CT score template that has excellent interrater reliability. ${ }^{12,13}$

\section{Statistical Analyses}

Data are first described using standard descriptive statistics. Wilcoxon rank-sum test and Spearman's correlation coefficient were then used to compare nonparametric data between prespecified groups. Any clot where the proximal or distal end could not be measured had length censored at $50 \mathrm{~mm}$ (36/104 patients). Our choice of nonparametric statistics based on ranks ensures that censoring of clot length at $50 \mathrm{~mm}$ and resultant imputation does not affect the results. Also, because our a priori hypothesis presumes the following causal chain "poor collaterals $\rightarrow$ (1) long clots $\rightarrow(2)$ hyperdense sign/GRE blooming," we tested the two associations separately using different statistical models. We also tested whether these associations were affected by time from stroke symptom onset to imaging. We used "censored linear regression" for model 1; our use of this technique takes into account censoring of the dependent variable data (i.e. clot length at $50 \mathrm{~mm}$ ). Collateral status and symptom onset to CT time were independent variables in model 1; this model also includes a two-way interaction term between collateral status and symptom onset to CT time. Collateral status was trichotomized into good (regional leptomeningeal score 17-20), intermediate (11-16), and poor (0-10) for these analyses. ${ }^{12}$ Model 2 a used logistic regression with the hyperdense sign as a dependent variable, clot length and onset to CT time as independent variables, and a two-way multiplicative interaction term between the independent variables. Model $2 b$ used logistic regression with GRE blooming as the dependent variable, clot length and onset to MR time as independent variables, and a two-way multiplicative interaction term between the independent variables. In all models, we report main effects when two-way multiplicative interactions are not significant. Interrater reliability $(\mathrm{n}=30)$ for clot length is reported using Spearman's correlation coefficient and for hyperdense sign and GRE blooming using unweighted kappa. A two-sided $\mathrm{p} \leq 0.05$ was considered statistically significant. All analyses were performed with STATA software (version 12.0; Stata Corp LP, College Station, TX, USA).

\section{Results}

\section{Baseline Demographics and Imaging Characteristics}

In 104 patients (mean age $65.1 \pm 12.28$ years, $56.7 \%$ male, median baseline National Institutes of Health Stroke Scale 13) included in the study, 50 patients had M1-MCA occlusion with intracranial ICA occlusion, whereas 54 had isolated M1-MCA clot. Median time from onset of stroke symptoms to CT imaging was 114 minutes (interquartile range [IQR] 114.5 minutes) and to MR imaging was 160 minutes (IQR 108 minutes). A good-quality baseline NCCT was present in 97 of 104 patients. The hyperdense sign on NCCT was seen in 45/97 (46.4\%) of patients on baseline NCCT. A GRE sequence at baseline was obtained in 76 of 104 patients. Blooming artifact on GRE was seen in 47/76 (61.8\%) of patients. Median clot length on CTA was $37.4 \mathrm{~mm}$ (IQR $29.6 \mathrm{~mm}$ ).

\section{Relationship Among Collateral Status, Clot Length, and Hyperdense Sign on NCCT and Blooming on GRE}

Patients with poor baseline collaterals had longer clots than those with intermediate collaterals or good collaterals (median clot length $49.4 \mathrm{~mm}$ vs $34.9 \mathrm{~mm}$ vs $20.5 \mathrm{~mm}$ respectively, $\mathrm{p}<0.001$ ) (Figures 3 and 4). Clots that are hyperdense on NCCT 


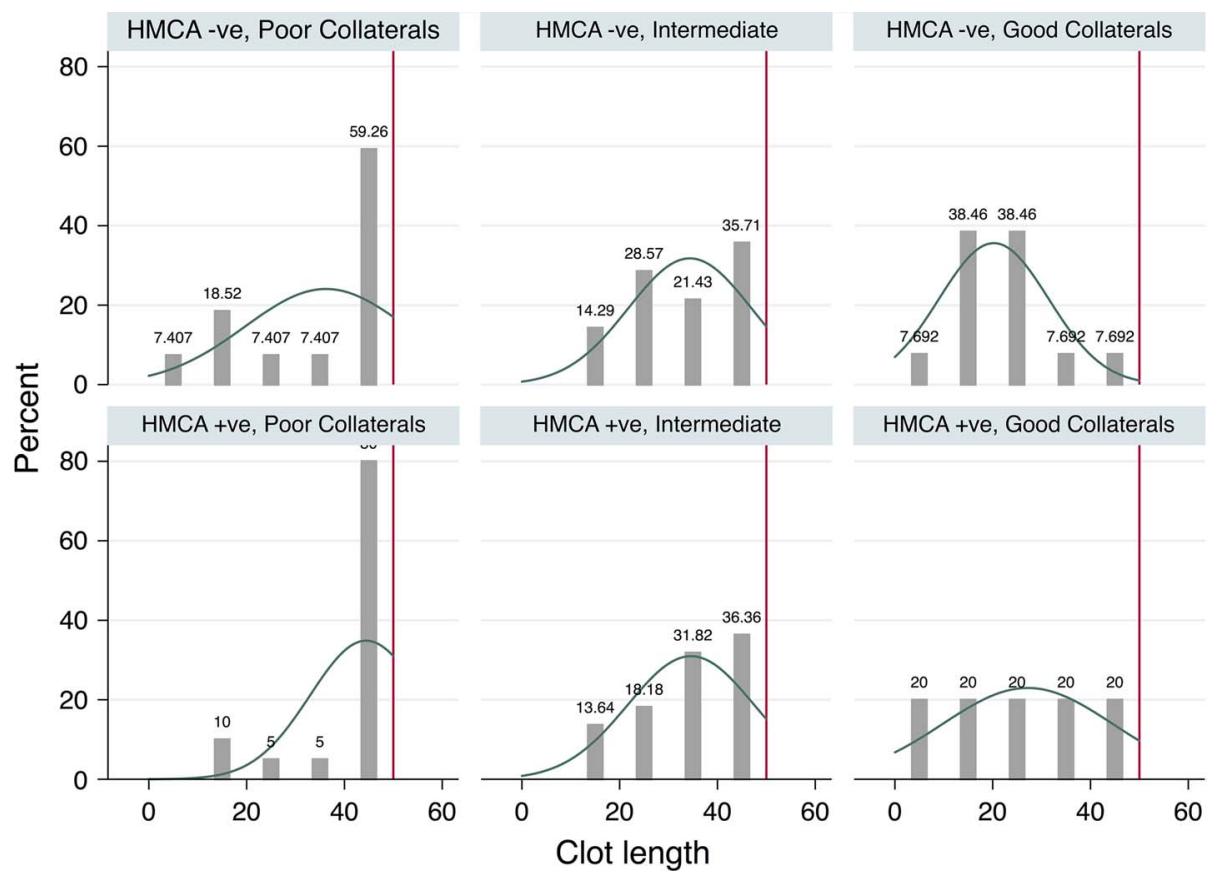

Figure 3: Relationship between the hyperdense sign on NCCT (hyperdense middle cerebral artery [HMCA]), collateral status, and clot length in our study. A left shift in the normal distribution of clot length occurs as collateral status improves, whereas a right shift in the same distribution is apparent in hyperdense clots $(H M C A+v e)$ when compared with clots that are not hyperdense (HMCA - ve). The data distribution suggests that longer clots are HMCA + ve and have poorer collaterals. Red line on the right indicates the threshold for censoring of clot length $(50 \mathrm{~mm})$.

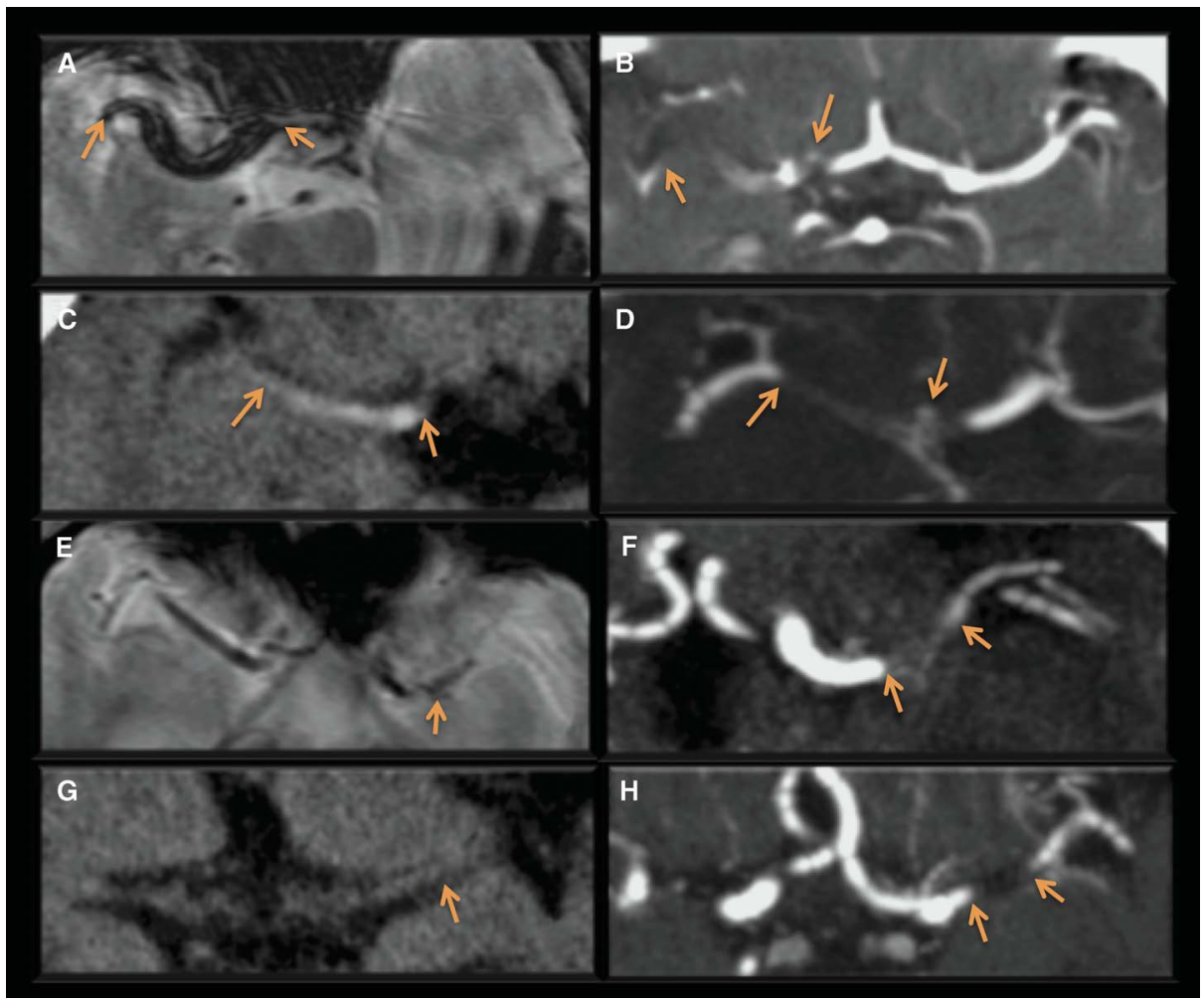

Figure 4: Panels on the left show presence (A) or absence (E) of blooming artifact on GRE and presence $(C)$ or absence $(G)$ of hyperdense sign on NCCT. Corresponding panels on the right show clot length on CTA. Longer clots on baseline CTA (corresponding panels on right) are associated with the hyperdense sign on NCCT and blooming artifact on GRE, whereas shorter clots are not. 

Table 1: Censored linear regression model (model 1) tests association between clot length and collateral status; logistic regres- sion models $2 \mathrm{a}$ and $2 \mathrm{~b}$ test association between hyperdense sign and clot length and between GRE blooming and clot length, respectively

\begin{tabular}{|c|c|c|c|c|}
\hline \multicolumn{5}{|c|}{ Model 1 (dependent variable: clot length) } \\
\hline Collateral status & Coefficient & SE & $95 \% \mathrm{CI}$ & p value \\
\hline Good $(n=19)$ & -25.4 & 5.89 & -37.11 to -13.75 & $<0.001$ \\
\hline Intermediate $(\mathrm{n}=36)$ & -10.6 & 4.84 & -20.05 to -0.86 & 0.033 \\
\hline Poor (reference) $(n=49)$ & - & - & - & - \\
\hline Stroke onset to CT time (minutes) & -0.001 & 0.01 & -0.02 to 0.02 & 0.908 \\
\hline \multicolumn{5}{|c|}{ Model 2a (dependent variable: hyperdense sign) } \\
\hline & Odds ratio & SE & $95 \% \mathrm{CI}$ & p value \\
\hline Clot length (per $1 \mathrm{~mm}$ ) & 1.03 & 0.01 & $1-1.06$ & 0.047 \\
\hline Stroke onset to CT time (minutes) & 1 & 0.001 & $1-1.001$ & 0.605 \\
\hline \multicolumn{5}{|c|}{ Model 2b (dependent variable: GRE blooming) } \\
\hline & Odds ratio & SE & 95\% CI & p value \\
\hline Clot length (per $1 \mathrm{~mm}$ ) & 1.04 & 0.02 & $1.01-1.08$ & 0.01 \\
\hline Stroke onset to MR time (minutes) & 1 & 0.001 & $1-1.001$ & 0.792 \\
\hline
\end{tabular}

CI, confidence interval; SE, standard error.

were longer (i.e. had a median clot length of $42.3 \mathrm{~mm}$ $[\mathrm{IQR}=24.2 \mathrm{~mm}])$ when compared with $29.5 \mathrm{~mm}([\mathrm{IQR}=33.9$ $\mathrm{mm}])$ for clots that were not hyperdense) $(\mathrm{p}=0.02)$ (Figures 3 and 4). Clots showing blooming artifacts on GRE were also longer (i.e. had a median clot length of $39.1 \mathrm{~mm}$ [IQR $=24 \mathrm{~mm}$ ] compared with $24.5 \mathrm{~mm}$ [IQR $=34.4 \mathrm{~mm}$ ] for clots without blooming on GRE) $(\mathrm{p}=0.005)$ (Figure 4$)$.

Model 1 (Table 1) shows that poor baseline collaterals are associated with longer clots. Clot length in patients with good collaterals is on average $25.4 \mathrm{~mm}$ less than in patients with poor collaterals ( $\mathrm{p} \leq 0.001$; main effect); clot length in patients with intermediate collaterals was on average $10.6 \mathrm{~mm}$ less than clot length in patients with poor collaterals. Symptom onset to CT time was not independently associated with clot length and did not modify or confound this association ( $\mathrm{p}=0.9$; interaction). Model $2 \mathrm{a}$ (Table 1) shows that longer clots were associated with presence of hyperdense sign $(p=0.047$; main effect), whereas model $2 \mathrm{~b}$ (Table 1) shows a similar association between clot length and blooming on GRE ( $p=0.01$; main effect). Symptom onset to CT or MR time is not independently associated with the dependent variable nor modifies or confounds this association (Table 1). Interrater reliability for clot length $(r=0.7)$, hyperdense sign $(\mathrm{k}=0.85)$, and GRE blooming $(\mathrm{k}=1)$ are very good.

\section{Discussion}

It is well-known that blood flow affects clot composition and that red clots form in low-pressure systems. ${ }^{4}$ These low-pressure systems include cardiac cul-de-sacs or venous systems., ${ }^{414}$ In contrast, "white clots" or platelet-rich clots form in highpressure systems, mainly in the arteries. ${ }^{4}{ }^{14}$ Previous studies have shown that the presence of the hyperdense sign on NCCT and blooming artifacts on GRE are due to RBC-rich "red clots.", RBC-rich clots have higher hemoglobin content when compared with platelet-rich or "white" clots and are therefore hyperdense on
NCCT and bloom on GRE. ${ }^{2,4}$ Our results show that longer clots on baseline CTA are more likely to be hyperdense on baseline NCCT and have blooming artifacts on GRE. We are also able to show that patients with poor baseline collaterals have longer clots on baseline CTA.

It is not intuitive to expect a very long sinuous original clot, possibly $>50 \mathrm{~mm}$ in length, to travel from a proximal source (e.g. heart) to the M1 MCA across the arch or aorta and the neck arteries. A more likely possibility is that longer clots seen in M1 MCA or ICA consist of (1) relatively smaller "original clot" that comes either from a proximal source or is formed in situ due to luminal disease and (2) new clot formation due to stasis of blood flow around this small, "original clot." We propose that poor collaterals and a lack of branching arteries near "original clot" create larger cul-de-sacs, which allow for stasis and new RBC-rich clot formation around the "original clot" (Figure 1). Good collaterals and branching arteries near the "original clot" may not create cul-de-sacs and resultant stasis (Figure 1). Collateral status and arterial branching patterns would therefore influence clot length and clot characteristics (Figures 1, 3, and 4). We were unable to analyze arterial branching patterns with the imaging technology at hand.

Studies trying to differentiate clot characteristics on imaging based on source of origin (i.e. a hyperdense clot from a cardiac/ venous source vs an iso- or hypodense clot from an arterial source [large arterial disease]) have not always shown robust correlations. $^{1-3,5,7,9,10}$ In our opinion, a reason for this lack of correlation is that imaging characteristics of clots are a reflection of not just "original clot" characteristics but also new clot formation locally around this original clot (Figure 1). The original clot itself may have varying signal characteristics on imaging depending on the original source of the clot, but because new clot formation can potentially constitute a significant portion of overall total clot burden, the predominant signal from the "total clot" may be heavily influenced by "new" clot constitution than that of the 


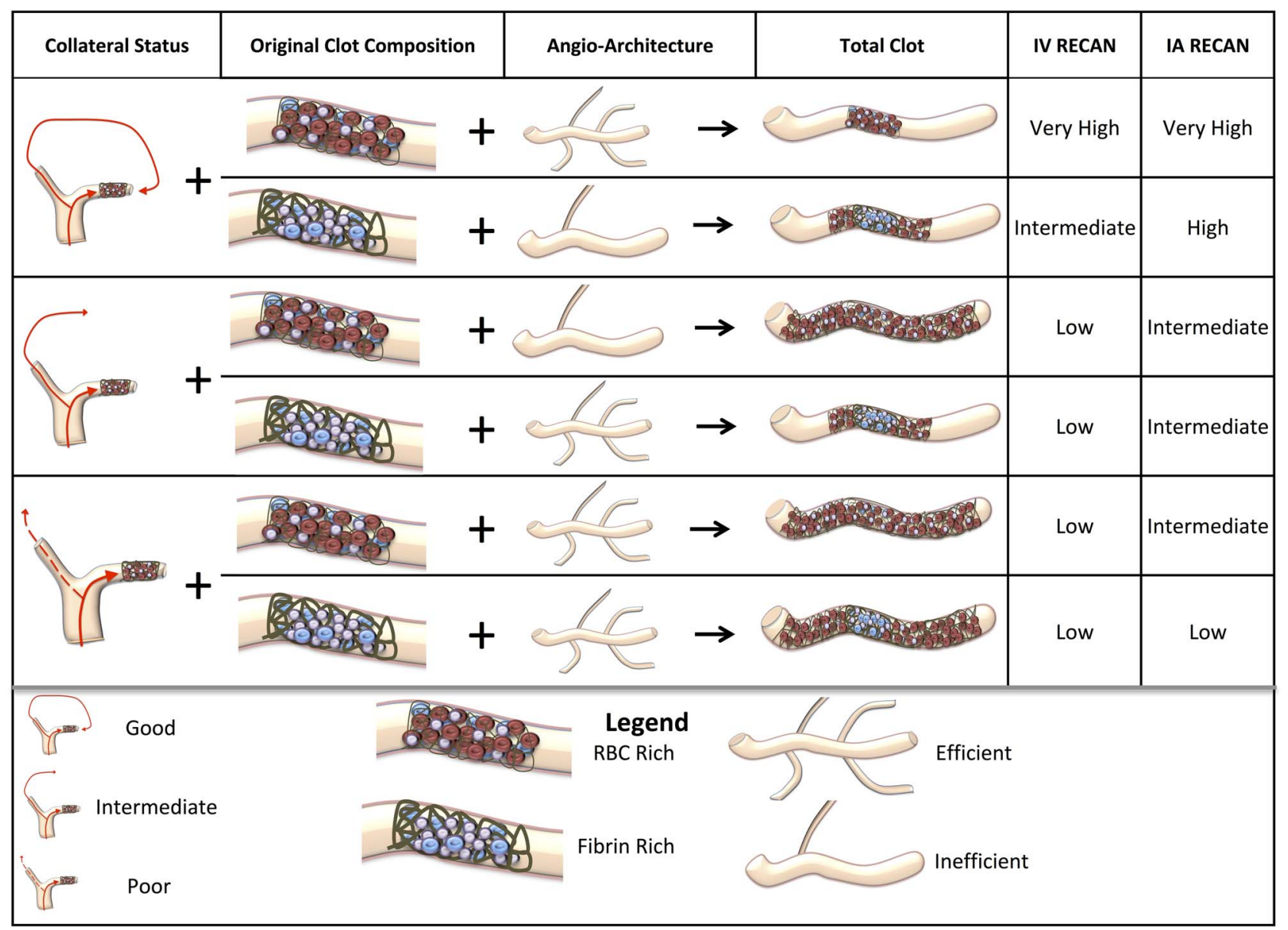

Figure 5: A theoretical explanation of clot characteristics on imaging and potential response to intravenous $t P A$ and intra-arterial therapy based on collateral status, original clot composition, and efficiency of angio-architecture. These are theoretical responses to intravenous tPA that have not been tested but are based on the previously mentioned parameters. All possible combinations are not included.

original clot. Nonetheless, our theory would suggest that some clots (with RBC-rich original clot) will have a homogenous signal throughout, whereas others (with fibrin-rich original clot) will have a heterogeneous signal with the fibrin-rich part of the clot being iso- or hypodense. Interestingly, as per our theory, the iso-/hypodense fibrin-rich part of the clot could be central or eccentric depending on proximal angio-architecture (Figures 1 and 5). This is corroborated by a scanning electron microscopic study of cerebral clots that reveals two distinct structural patterns within intracranial clots: (1) thrombus displaying advanced maturity where all thrombotic elements are densely integrated and individually not discernible and (2) thrombus displaying trapped RBCs and fibrin in a loose cross-linking form. ${ }^{6}$ Different patients had varying proportions of these two structural patterns within their clots. ${ }^{6}$

Previous studies have shown that patients with good collaterals have high recanalization and poor collaterals have low recanalization rates with intra-arterial therapy. ${ }^{15,16}$ Potential explanations for this relationship between good collaterals and recanalization include good retrograde collateral filling providing more access to thrombolytics at the distal end of clot (in patients given intravenous tPA along with intra-arterial therapy) and robust collaterals dissolving clot fragments in the distal vasculature. ${ }^{15,17}$ Our theory suggests that patients with poor collaterals and/or inefficient angio-architecture will have longer clots extending into pial arteries; increasing clot burden will therefore reduce recanalization with both intravenous IPA and intra-arterial therapy, more so with intravenous tPA. Original clot composition (whether RBC- or fibrin-rich) will interact with collateral status and angio-architecture to determine overall clot characteristics and the probability of recanalization with intravenous tPA and intraarterial therapy. Figure 5 proposes several theoretical combinations of collateral status, angio-architecture, and original clot composition with resultant imaging characteristics on NCCT and GRE MRI and probability of recanalization with intravenous tPA and intra-arterial therapy.

Our analysis shows no correlation between clot length and time from stroke symptom onset to imaging. Attenuation characteristics of a clot can reduce over time as clots change composition from RBC-rich clots to more organized, fibrin-rich variants. ${ }^{18}$ Nonetheless, this evolution of attenuation characteristics invariably happens after 6 hours of clot formation. ${ }^{18}$ Because most of our patients are imaged within 6 to 8 hours of stroke symptom onset, we could not find any association between hyperdense sign/GRE blooming and time from stroke symptom onset to CT or MRI.

A limitation of our study is that we were not able to quantitate efficiency of angio-architecture around the "original clot." Unlike 
collateral status within the cerebral arterial tree, angio-architecture efficiency cannot be measured directly on CTA. We need in vitro studies to study the role of angio-architecture in clot formation. We also have not attempted a detailed analysis of heterogeneity of clot signal on NCCT nor tested the ability of our theory to predict recanalization rates or final clinical outcomes with intravenous tPA and intra-arterial therapy. To attempt this, we will need thinslice NCCT scans and a substantially larger sample size. Future studies should aim to quantify angio-architecture and predict recanalization based on collateral status and angio-architecture.

\section{CONCLUSION}

We demonstrate that longer clots are more likely to be hyperdense on NCCT, and bloom more on GRE MRI. We use this information to propose a coherent new theory of clot formation in the cerebral arterial tree and the role of collaterals and angioarchitecture in determining clot size and composition. In addition, our theory offers a template for understanding clot lysis with intravenous tPA and intra-arterial therapy within the cerebral arterial tree. Finally, our study offers a theoretical alternative explanation for why patients with good collaterals are more likely to benefit clinically with intravenous tPA and intra-arterial therapy.

\section{DisClosures}

MG has received honoraria from and served as a consultant to Covidien; was a co-principal investigator for the ESCAPE Trial, SWIFT Prime, but received no financial compensation; and has a licensing agreement with GE, but no financial compensation was received. $\mathrm{AD}$ has received honoraria from and served as a consultant to Covidien. BKM is a researcher for and has received research support from Canadian Institute of Health Research, HSFC, Alberta Innovates Health Solutions, University of Calgary, and Hotchkiss Brain Institute. EQ, SIS, SM, MA, ME, Cd'E, AQ, and JP have nothing to disclose.

\section{ACKNOWLEDGMENT}

We acknowledge Dr. Sajobi's advice on the statistical analyses in the manuscript.

\section{REFERENCES}

1. Riedel $\mathrm{CH}$, Jensen $\mathrm{U}$, Rohr A, et al. Assessment of thrombus in acute middle cerebral artery occlusion using thin-slice nonenhanced computed tomography reconstructions. Stroke. 2010;41: $1659-1664$
2. Liebeskind DS, Sanossian N, Yong WH, et al. CT and MRI early vessel signs reflect clot composition in acute stroke. Stroke. 2011;42:1237-43.

3. Bastianello S, Pierallini A, Colonnese C, et al. Hyperdense middle cerebral artery CT sign. Comparison with angiography in the acute phase of ischemic supratentorial infarction. Neuroradiology. 1991;33:207-11.

4. Minnerup J, Kleinschnitz C. Visualization of clot composition in ischemic stroke: do we get what we see? Stroke. 2011;42: 1193-94.

5. Kimura K, Iguchi Y, Shibazaki K, Terasawa Y, Aoki J, Matsumoto N. The presence of a right-to-left shunt is associated with dramatic improvement after thrombolytic therapy in patients with acute ischemic stroke. Stroke. 2009;40:303-5.

6. Mehta BP, Nogueira RG. Should clot composition affect choice of endovascular therapy? Neurology. 2012;79(13 Suppl 1): S63-7.

7. Marder VJ, Chute DJ, Starkman S, et al. Analysis of thrombi retrieved from cerebral arteries of patients with acute ischemic stroke. Stroke. 2006;37:2086-93.

8. Almekhlafi MA, Hu WY, Hill MD, Auer RN. Calcification and endothelialization of thrombi in acute stroke. Ann Neurol. 2008;64:344-8.

9. Barber PA, Demchuk AM, Hudon ME, Pexman JHW, Hill MD, Buchan AM. Hyperdense sylvian fissure MCA "dot" sign: a CT marker of acute ischemia. Stroke. 2001;32:84-8.

10. Cho KH, Kim JS, Kwon SU, Cho AH, Kang DW. Significance of susceptibility vessel sign on $\mathrm{T} 2 *$-weighted gradient echo imaging for identification of stroke subtypes. Stroke. 2005;36:2379-83.

11. Tsushima Y, Aoki J, Endo K. Brain microhemorrhages detected on T2*-weighted gradient-echo MR images. AJNR Am J Neuroradiol. 2003;24:88-96.

12. Menon BK, Smith EE, Modi J, et al. Regional leptomeningeal score on CT angiography predicts clinical and imaging outcomes in patients with acute anterior circulation occlusions. AJNR Am J Neuroradiol. 2011;32:1640-5.

13. Menon BK, Smith EE, Coutts SB, et al. Leptomeningeal collaterals are associated with modifiable metabolic risk factors. Ann Neurol. 2013;74:241-8.

14. Brogan GX, Jr. Bench to bedside: pathophysiology of acute coronary syndromes and implications for therapy. Acad Emerg Med. 2002;9:1029-44.

15. Bang OY, Saver JL, Kim SJ, et al. Collateral flow predicts response to endovascular therapy for acute ischemic stroke. Stroke. 2011;42:693-9.

16. Liebeskind DS, Tomsick TA, Foster LD, et al. Collaterals at angiography and outcomes in the interventional management of stroke (IMS) III Trial. Stroke. 2014;45:759-64.

17. Caplan LR, Hennerici M. Impaired clearance of emboli (washout) is an important link between hypoperfusion, embolism, and ischemic stroke. Arch Neurol. 1998;55:1475-82.

18. Kirchhof K, Welzel T, Mecke C, Zoubaa S, Sartor K. Differentiation of white, mixed, and red thrombi: value of CT in estimation of the prognosis of thrombolysis phantom study. Radiology. 2003; 228:126-30. 